In: Proceedings of the Fifth workshops on Enabling Technologies:

Infrastructure for Collaborative Enterprises (WETICE'96), Stanford,

California, June 19-21, 1996. IEEE Computer Society Press. Pp232-237.

\title{
Independent Validation of Specifications: A coordination headache
}

\author{
Steve Easterbrook \\ steve@atlantis.ivv.nasa.gov \\ John Callahan \\ Software Research Lab \\ NASAMWU IV\&V Facility \\ Fairmont, WV 26554
}

\section{Abstract}

Large, complex projects face significant barriers to coordination and communication due to continuous, rapid changes during a project's lifecycle. Such changes must be tracked, analyzed, and reconciled to ensure high-quality in the end-product, otherwise problems may be get lost or ignored in the overall complexity. We report on "work-inprogress" in the study of coordination problems between two independent, separate groups: software development and software analysis. We have begun to construct a taxonomy of coordination problem, which we illustrate with two scenarios. We briefly describe current attempts to introduce incremental improvements to coordination problems in such projects via World-Wide-Web tools. Based on actual project experiences, we plan to deploy such tools in a non-intrusive fashion to improve coordination and communication between software development groups.

\section{Introduction}

This paper describes some of the coordination problems faced in the verification and validation (V\&V) of large software systems. In particular, we examine the process of Independent V\&V (or IV\&V). a practice used on large. safety-critical software systems in the defense and aerospace industries. IV\&V faces many of the same coordination problems as any large development project. and introduces many problems of its own. In particular, IV \&V requires two organizations with conflicting goals to cooperate to satisfy a single customer. Not only is there a lack of suitable tools to support the coordination needed. but often even the infrastructure in which such tools would be used is missing. We will illustrate some of the problems faced by IV\&V through two scenarios. and describe our initial work on the development of web-based tools that address the problems.
Although we focus on IV\&V, many of the coordination problems are found in any large project. In many projects, coordination problems surface merely as tensions and frustrations. perhaps leading to schedule and budget problems. In an IV\&V process. the coordination crosses organizational boundaries. This makes it harder for the people involved to find ways to work around any lack of cooperation. Hence, the problems are more visible. and easier to study.

Within the IV\&V process, we concentrate on the analysis of requirements specifications. This is a deliberate choice: independent analysis of requirements specifications has the potential for the biggest impact of any IV\&V activity. The quality of the analysis of design and implementation and the rigor of testing depend to some extent on the availability of good quality specifications.

In this paper, we identify the interdependence of large volumes of documentation as a key problem in software specification. Any change to one part of a specification may have many small impacts ("ripples of influence" [6]) throughout the documentation, which may be hard to track down. Certainly this kind of problem was an important factor in the fatal decision to launch Challenger [1]. The mechanical problem that caused the blast was well known before the accident; however, faitures in tracking the problem and in keeping all documentation consistent led to faulty decision making by NASA managers.

In this paper. we outline the IV\&V process and identify a number of coordination problems that we have observed in actual IV\&V processes. Specifically, we draw on our informal observations of and interactions with IV\&V personnel working on the Space Station and the Earth Sciences Data and Information System (ESDIS) projects. We present two scenarios. which reveal the extent to which coordination problems can reduce the effectiveness of IV\&V. We then describe our initial wat with the World Wide Web to provide infrastructure and tools to overcame some of these problems. 